\title{
Article \\ Nanocarriers Based on Gold Nanoparticles for Epigallocatechin Gallate Delivery in Cancer Cells
}

\author{
Lídia Cunha ${ }^{1,2}$, Sílvia Castro Coelho ${ }^{1,2, *(\mathbb{D}}$, Maria do Carmo Pereira ${ }^{1,2} \mathbb{C}$ and Manuel A. N. Coelho ${ }^{1,2}$ \\ 1 LEPABE-Laboratory for Process Engineering, Environment, Biotechnology and Energy, \\ Faculty of Engineering, University of Porto, Rua Dr. Roberto Frias, 4200-465 Porto, Portugal; \\ up201406702@edu.fe.up.pt (L.C.); mcsp@fe.up.pt (M.d.C.P.); mcoelho@fe.up.pt (M.A.N.C.) \\ 2 ALiCE-Associate Laboratory in Chemical Engineering, Faculty of Engineering, \\ University of Porto, Rua Dr. Roberto Frias, 4200-465 Porto, Portugal \\ * Correspondence: silviac@fe.up.pt; Tel.: +351-225082262; Fax: +351-225081449
}

check for

updates

Citation: Cunha, L.; Coelho, S.C.; Pereira, M.d.C.; Coelho, M.A.N. Nanocarriers Based on Gold Nanoparticles for Epigallocatechin Gallate Delivery in Cancer Cells. Pharmaceutics 2022, 14, 491. https://doi.org/10.3390/ pharmaceutics14030491

Academic Editor: Carlos Alonso-Moreno

Received: 31 December 2021 Accepted: 21 February 2022 Published: 24 February 2022

Publisher's Note: MDPI stays neutral with regard to jurisdictional claims in published maps and institutional affiliations.

Copyright: (C) 2022 by the authors. Licensee MDPI, Basel, Switzerland. This article is an open access article distributed under the terms and conditions of the Creative Commons Attribution (CC BY) license (https:// creativecommons.org/licenses/by/ $4.0 /)$.

\begin{abstract}
Gold nanoparticles (AuNPs) are inorganic and biocompatible nanovehicles capable of conjugating biomolecules to enhance their efficacy in cancer treatment. The high and reactive surface area provides good advantages for conjugating active compounds. Two approaches were developed in this work to improve the Epigallocatechin-3-gallate (EGCG) antioxidant efficacy. AuNPs were synthesized by reducing gold salt with chitosan. One other nanosystem was developed by functionalizing AuNPs with cysteamine using the Turkevitch method. The physico-chemical characterization of EGCG conjugated in the two nanosystems-based gold nanoparticles was achieved. The in vitro toxic effect induced by the nanoconjugates was evaluated in pancreatic cancer cells, showing that encapsulated EGCG keeps its antioxidant activity and decreasing the BxPC3 cell growth. A significant cell growth inhibition was observed in 50\% with EGCG concentrations in the range of 2.2 and $3.7 \mu \mathrm{M}$ in EGCG-ChAuNPs and EGCG-Cyst-AuNPs nanoconjugates, respectively. The EGCG alone had to be present at $23 \mu \mathrm{M}$ to induce the same cytotoxicity response. Caspase-3 activity assay demonstrated that the conjugation of EGCG induces an enhancement of BxPC3 apoptosis compared with EGCG alone. In conclusion, AuNPs complexes can be used as delivery carriers to increase EGCG antioxidant activity in cancer tissues.
\end{abstract}

Keywords: drug delivery systems; gold nanoparticles; epigallocatechin gallate; antioxidant activity; pancreatic cancer cells

\section{Introduction}

Gold nanoparticles are vectors that present the capacity to target biocompounds without loss of their activity and overcome the drawbacks associated with the conventional agents used in cancer treatment. The low bioavailability, stability and half-life time are the main limitations of biocompounds [1,2]. The concept of the presented study is based on the delivery of an antioxidant agent using gold nanoparticles (AuNPs). AuNPs are inorganic vehicles that present unique physical, chemical and optical characteristics $[3,4]$. Their reactive surface, easy conjugation and biocompatibility provide high loading capacity for drug entrapment and improve its stability in several biomedical applications [3].

Epigallocatechin-3-gallate (EGCG) is the chosen antioxidant in this study. It is a natural green tea compound that possesses powerful antioxidant and anticancer activities [5-8]. This molecule presents a short half-life, low stability and low bioavailability [6,9]. Several studies reported a significant EGCG effect verified on cancer cells lines and animal model by angiogenesis, apoptosis, and transcription factor [5]. In recent years, AuNPs have been used as a system to protect and deliver drugs/active molecules in situ [5]. The excellent capacity of nanoparticles to modify their surface with targeting ligands allows improving their accumulation in tumors compared to free molecules $[6,10]$. The delivery of molecules to tumor occurs by passive targeting due to the enhanced permeability and retention (EPR) 
effect [3]. Safwat et al. demonstrated the good potential of AuNPs to increase anticancer effect of EGCG in Ehrlich ascites carcinoma-bearing mice [6]. Sanna et al. suggested that $25 \mathrm{~nm}$ stable EGCGAuNPs present more antioxidant activity when compared with EGCG alone [11]. Additionally, the in vitro cell growth study of EGCGAuNPs assessed against human neuroblastoma SH-SY5Y-derived cells found high toxicity of the nanosystem compared with EGCG alone. Mostafa et al. reported the $35 \mathrm{~nm}$ EGCGAuNPs with high toxicity on hepatocellular carcinoma HepG2 cells compared with EGCG alone [12]. The let7a and miR-34a expression in the cells were analyzed, demonstrating their high expression levels after $72 \mathrm{~h}$ of incubation with EGCGAuNPs.

The aim of this study is to develop a drug-delivery nanosystems-based AuNPs with optimal size range diameters (between 100-200 nm) promoting a high cell uptake rate $[13,14]$. The nanoparticles might be internalized via clathrin- or caveolin-mediated endocytosis [14]. The developed nanoformulations presented opposite charges to incorporate EGCG. The strategy was to synthesize both AuNPs using chitosan as the reducing agent and cysteamine as capping agent $[15,16]$. Chitosan was chosen to improve the nanosystem biocompatibility $[17,18]$. Cysteamine was selected to functionalize gold nanoparticles in order to increase the therapeutic effect of the nanosystem $[19,20]$. The enhancement of the nanosystems efficacy was evaluated. The behavior application of the chitosan-gold nanoparticles (ChAuNPs) and cysteamine-Au NPs (CystAuNPs) as the delivery vehicles of EGCG were studied using the pancreatic adenocarcinoma BxPC3 cell line. EGCG-based AuNPs might increase the solubility and bioavailability of the active compound, prolonging its circulation time and induce to higher levels of inhibition [21].

ChAuNPs were produced by a green synthesis method with chitosan $(\mathrm{Ch})$ as reducing agent. Chitosan is a natural cationic polysaccharide derived from partial chitin deacetylation. It is constituted by $\beta-(1-4)$-linked D-glucosamine and N-acetyl-D-glucosamine randomly distributed within the polymer $[17,22,23]$. Ch is a biocompatible and nontoxic polysaccharide. It presents low allergenicity and biodegradability properties [3,24]. Bhattarai et al. reported the production of N-acylated chitosan-stabilized AuNPs for applications in physiological conditions, exhibiting the advantages of non-acylated chitosan [25]. Several studies demonstrated the antitumor activity of chitosan in in vitro and in vivo models $[22,26]$. Lin et al. suggested the significant anti-tumorigenesis effect of chitosan against gastric and colon cancer cells [26]. Safer et al. prepared and characterized $414 \mathrm{~nm} \mathrm{Au/Ch/EGCG} \mathrm{nanoconjugates} \mathrm{that} \mathrm{might} \mathrm{be} \mathrm{used} \mathrm{in} \mathrm{the} \mathrm{hepatic} \mathrm{fibrosis} \mathrm{disorder}$ treatment [27].

AuNPs were synthetized by the Turkevich method and then functionalized with cysteamine CystAuNPs [2]. Some studies suggested the positive therapeutic effects for cancer $[8,19,28]$. Fujisawa et al. reported cysteamine, an antioxidant aminothiol, to inhibit metastasis of pancreatic cancer [19]. Wan et al. showed the increase in doxorubicin chemotherapeutic effect by the autophagy-modulatory role of cysteamine in cancer cells [28]. Inano et al. suggested cysteamine as an inhibitor of estrogen receptor development of mammary tumors in rats [29]. Rubin et al. validated that cysteamine inhibits pancreatic cancer [20].

\section{Materials and Methods}

\subsection{Materials}

Epigallocatechin gallate (MW $458.372 \mathrm{~g} / \mathrm{mol}$ ) was obtained from Taiyo Kagaku. Chitosan (MW $250 \mathrm{kDa}$ and degree of deacetylation > 93\%) was acquired from Altakitin (Lisbon, Portugal). Trisodium hydroxide and tetrachloroauric (III) acid ( $\mathrm{HAuCl}_{4} ; 99.99 \%$ trace metals basis, $30 \mathrm{wt} \%$ in dilute $\mathrm{HCl}$ ), acetic acid, dimethyl sulfoxide (DMSO), NHydroysulfosuccinimide sodium salt (MW $217.13 \mathrm{~g} / \mathrm{mol}$ ), n-(3-Dimethylaminopropyl)-N'ethyliarbodiimied hydrochloride (MW $191.70 \mathrm{~g} / \mathrm{mol}$ ), tween 80, sulforhodamine B (SRB), and trypan blue were purchased from Sigma-Aldrich (Darmstadt, Germany). Phosphate buffered saline (PBS: 0.01 M, pH 7.4) was purchased from Fluka (München, Germany). The antioxidant kit assay kit (Cayman Chemical Company) was obtained from Cayman 
Chemical CompanyMaster-InVitro (Barcelona, Spain). Regenerated cellulose dialysis membrane $(8-10 \mathrm{kD})$ was acquired from Spectra/Por ${ }^{\circledR}$. Amicon ultra ( $3 \mathrm{k}$, Merck KgaA) was obtained from Millipore S.A.S. (Molsheim Cedex, France). Cysteamine (MW 77.15 g/mol) was obtained from Selleck Chemicals LLC (Houston, TX, USA). BxPC-3 CRL-1687 cells was purchased from LGC standards, S.L.U. (Barcelona, Spain). Fetal bovine serum (FBS), phosphate-buffered saline (PBS), trypsin, Roswell Park Memorial Institute Medium (RPMI 1640 Medium) were obtained from Invitrogen Co. (Scotland, UK).

\subsection{Conjugation of EGCG with Gold Nanoparticles Synthetized with Chitosan (EGCG-ChAuNPs)}

An aqueous solution of acid chloroauric $(1.0 \mathrm{mM}, 1.0 \mathrm{~mL})$ was mixed with a diluted solution of chitosan $(5.0 \mathrm{~mL}, 0.32 \mathrm{w} / \mathrm{v} \%)$ under magnetic stirring [30]. The mixture was heated for $30 \mathrm{~min}$ until a red suspension was obtained and ChAuNPs prepared. The concentration of the colloidal suspension ChAuNPs was $31.8 \mathrm{nM}$ as calculated by the Lambert-Beer Law with a molar absorptivity of the AuNPs of $2.33 \times 10^{8} \mathrm{M}^{-1} \mathrm{~cm}^{-1}$ (plasmon resonance band at $526 \mathrm{~nm}$ ) [19,31].

A total of $2.0 \mathrm{~mL}$ ChAuNPs were then conjugated with $1.0 \mathrm{~mL}$ of $0.27 \mathrm{mM}$ EGCG via carbodiimide-mediated cross-linking (EDC/NHSS) with a molar ratio EGCG: EDC/NHSS of 1:0.5, at room temperature (RT) for one hour. The final solution was centrifuged at $12,000 \mathrm{rpm}$ during $8 \mathrm{~min}$ to remove the excess of EGCG. The concentration of the EGCGChAuNPs was 14.5 nM (Lambert-Beer Law, Figure S1A).

\subsection{Conjugation of EGCG with CystAuNPs (EGCG-CystAuNPs)}

AuNPs were synthesized by the Turkevitch method. An aqueous solution of acid chloroauric ( $25 \mathrm{~mL}$ water, $17.2 \mu \mathrm{L} \mathrm{HAuCl}_{4}$ ) was mixed with a diluted solution of sodium citrate $(2.5 \mathrm{~mL}, 0.03 \mathrm{~g})$. The reaction time was $10 \mathrm{~min}$ under magnetic stirring and heating. Then, the AuNPs were functionalized with cysteamine with a molar ratio cysteamine: AuNPs of 1:1 [15,32]. The solution was stirred for $30 \mathrm{~min}$ at RT. The final nanoparticles concentration was $11.5 \mathrm{nM}$.

A total of $1.0 \mathrm{~mL}$ of $0.27 \mathrm{mM}$ EGCG was conjugated with $5.0 \mathrm{~mL}$ of CystAuNPs via EDC/NHSS coupling reaction (with a molar ratio EGCG: EDC/NHSS of 1:01), under magnetic stirring, at room temperature. After, tween $80(0.11 \mathrm{~g})$ was added to the nanoparticles to enhance their stability, reacting during $15 \mathrm{~min}$. The final solution was centrifuged at $12,000 \mathrm{rpm}$, for $8 \mathrm{~min}$ to remove the unbound EGCG. The estimated EGCG-CystAuNPs concentration was $1.36 \mathrm{nM}$ (Figure S1B).

\subsection{Dynamic Light Scattering}

The hydrodynamic diameter (dynamic light scattering, DLS) and the zeta potential (laser Doppler velocimetry, LDV) of the nanoparticles in suspension were investigated using a Zetasizer Nano ZS (Malvern Instruments Ltd., Malvern, UK), at $25^{\circ} \mathrm{C}$. The size distribution was determined in a $12 \mathrm{~mm}$ square polystyrene cuvette (DTS 1060, Sarstedt, Nümbrecht, Germany), at a scattering angle of $173^{\circ}$.

\subsection{Transmission Electron Microscopy (TEM)}

TEM images were obtained using a Jeol JEM-1400, JEOL operated at $60 \mathrm{kV}$. A total of $5 \mu \mathrm{L}$ of sample was placed on a carbon formvar-coated grid for $5 \mathrm{~min}$ and washed to remove the excess.

\subsection{UV-Vis Spectroscopy}

UV-Vis absorption spectra of the samples-AuNPs, ChAuNPs, CystAuNPs, EGCGChAuNPs and EGCG-CystAuNPs—-were analyzed by Shimadzu UV-1700 PharmaSpec spectrophotometer, at RT. 


\subsection{EGCG Encapsulation Efficiency}

The UV-Vis absorption spectra of the supernatant were determined, using Shimadzu UV-1700 PharmaSpec spectrophotometer, and the EGCG encapsulation efficiency (EE) of the nanoconjugates was calculated using Equation (1):

$$
\mathrm{EE}(\%)=\frac{\text { Total EGCG in suspension }- \text { EGCG in supernatant }}{\text { Total EGCG in suspension }} \times 100
$$

\subsection{Attenuated Total Reflectance-Fourier Transform Infrared Spectroscopy (ATR-FTIR)}

The suspensions of ChAuNPs, EGCG-ChAuNPs, CystAuNPs and EGCG-CystAuNPs were evaluated by ATR-FTIR spectroscopy with an ALPHA FTIR Spectrometer (Bruker) in the spectral range $4000-400 \mathrm{~cm}^{-1}$, resolution of $4 \mathrm{~cm}^{-1}$ and 64 scans, at RT.

\subsection{In Vitro Release Studies}

EGCG-ChAuNPs and EGCGCys-AuNPs release profiles were achieved in DI water. The samples were incubated with constant magnetic stirring, at $37{ }^{\circ} \mathrm{C}$ in regenerated cellulose dialysis membranes. Figure S2 displays the lowest and the highest in vitro typical release spectra corresponding to $\mathrm{C} / \mathrm{CEGCG}$.

\subsection{Antioxidant Assay}

The antioxidant capacity of the systems EGCG-ChAuNPs and EGCG-CysAuNPs was measured using the Cayman's antioxidant assay kit.

\subsection{Cell Culture}

Human pancreatic cancer cell lines (BxPC3) were maintained in an RPMI medium, complemented with $10 \%$ FBS, at $37^{\circ} \mathrm{C}$ under $5 \% \mathrm{CO}_{2}$ humidified atmosphere.

\subsection{In Vitro Cytotoxicity Evaluation}

The effects of the nanosystems and EGCG alone on the BxPC3 cell growth were assessed by the Sulforhodamine B (SRB) method.

A total of 1000 BxPC3 cells per well were seeded under $5 \% \mathrm{CO}_{2}$ humidified atmosphere, at $37^{\circ} \mathrm{C}$. Cells were treated with the control samples and samples. The controls are CystAuNPs and ChAuNPs at the concentrations ranging between 0 and $1.0 \mathrm{nM}$ for $24 \mathrm{~h}$. The samples are EGCG, EGCG-CystAuNPs and EGCG-ChAuNPs with EGCG concentrations between 2.0 and $25 \mu \mathrm{M}$. Following the $24 \mathrm{~h}$ incubation, the cells fixated with $10 \%$ TCA for $1 \mathrm{~h}$ on ice, were washed and stained with $50 \mu \mathrm{L} \mathrm{SRB}$ dye for $30 \mathrm{~min}$. After, $1 \%$ acetic acid was used to the wash the cells. The samples were dried, and the protein-bound stain was solubilized with $10 \mathrm{mM}$ Tris solution. The SRB absorbance was evaluated at $560 \mathrm{~nm}$ using the PowerWave microplate reader. Figure S5 exhibits the lowest and the highest cell growth results of the BxPC3 after $24 \mathrm{~h}$ incubation with the nanoparticles alone. Figure S6 shows the lowest and the highest growth results after incubation with free EGCG, EGCGAuChNPs and EGCG-CysAuNPs. The IC50 concentrations of EGCG, EGCG-CystAuNPs and EGCG-ChAuNPs were estimated.

\subsection{Caspase-3 Activation Assay}

The nanosystems were dissolved in PBS buffer and incubated with BxPC3 cells at an EGCG concentration of $5 \mu \mathrm{M}$ for $24 \mathrm{~h}$. The toxicity of the samples was analyzed by the activation of caspase-3. Briefly, confluent BxPC3 were incubated with EGCG, EGCGChAuNPs and EGCG-CystAuNPs for $24 \mathrm{~h}$. The cells were tripsinized and centrifuged. The pellet of cells was lysed in $100 \mu \mathrm{L}$ hypotonic lysis buffer. To estimate caspase- 3 activation, $40 \mu \mathrm{L}$ of the lysate solution was used to achieve the total cellular protein concentration at $595 \mathrm{~nm}$. Figure 57 exhibits the lowest and the highest results obtained for caspase- 3 activation assay. 


\subsection{Statistical Analysis}

Three independent experiments were measured. Statistical significance $(p<0.05)$ was determined by the Student's $t$-test.

\section{Results and Discussion}

TEM images (Figure 1) present the ChAuNPs and CystAuNPs formulated. A polydisperse ChAuNPs suspension was synthesized through the reduction in gold salt by chitosan presenting a spherical shape (Figure 1A). These results corroborate intensity distribution graph and size analyzed by DLS measurements (Table 1). CystAuNPs were prepared by the functionalization with cysteamine of gold nanoparticles synthesized by the Turkevitch method [33]. CystAuNPs (Figure 1B) have mainly spherical shapes.

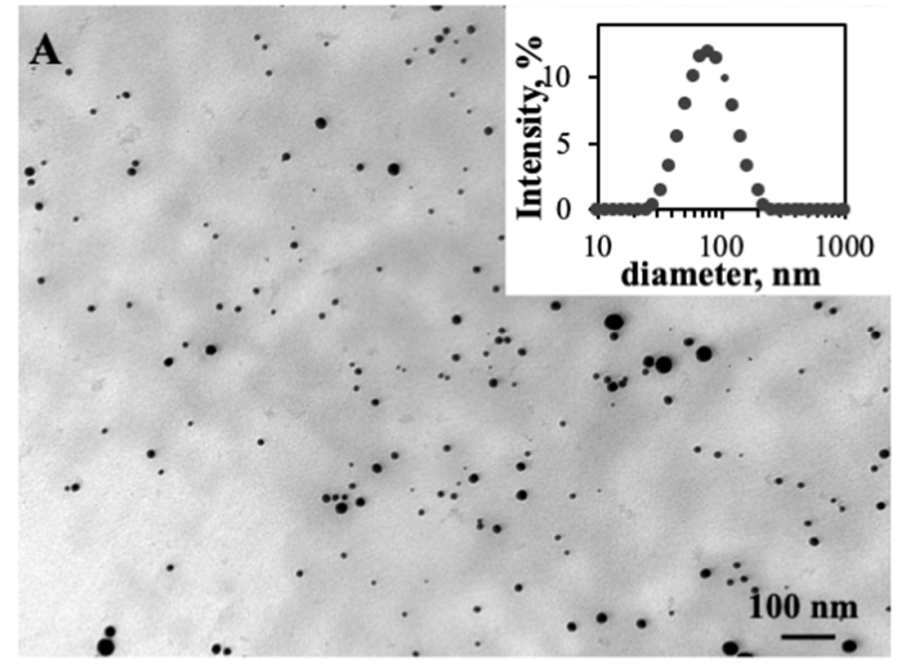

B
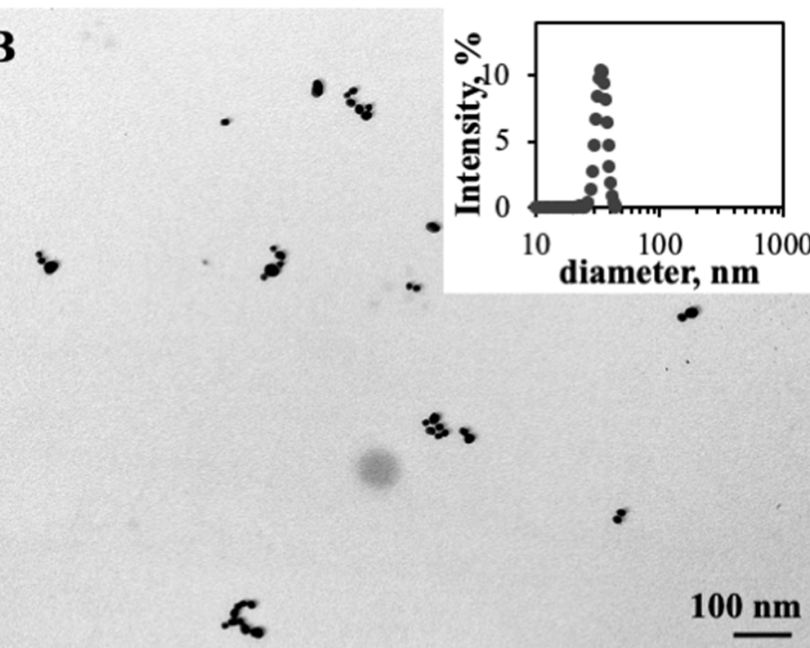

$100 \mathrm{~nm}$

Figure 1. TEM image of: (A) ChAuNPs and (B) CystAuNPs. The scale bar is $100 \mathrm{~nm}$.

Table 1. Hydrodynamic diameter, polydispersity index (PdI) and zeta potential of AuNPs, CystAuNPs, EGCG-CystAuNPs, ChAuNPs, EGCG-ChAuNPs.

\begin{tabular}{cccc}
\hline Samples & Size, $\mathbf{n m}$ & PdI & Zeta Potential, $\mathbf{m V}$ \\
\hline AuNPs & $37 \pm 1$ & 0.2 & $-33 \pm 2$ \\
CystAuNPs & $54 \pm 9$ & 0.6 & $-37 \pm 3$ \\
EGCG-CystAuNPs & $111 \pm 1$ & 0.2 & $-24 \pm 1$ \\
ChAuNPs & $86 \pm 16$ & 0.2 & $22 \pm 5$ \\
EGCG-ChAuNPs & $125 \pm 13$ & 0.3 & $36 \pm 6$
\end{tabular}

AuNPs: gold nanoparticles; ChAuNPs: chitosan-gold nanoparticles; EGCGChAuNPs: conjugated chitosan-gold nanoparticles with EGCG; CystAuNPs: gold nanoparticles functionalized with cysteamine; EGCGCystAuNPs: Conjugated CystAuNPs with EGCG $(n=3)$.

The hydrodynamic diameters and the zeta potential values of the nanoparticles are summarized in Table 1. A total of $86 \pm 16 \mathrm{~nm}$ ChAuNPs were obtained with a zeta potential of approximately $22 \mathrm{mV}$, suggesting good stability of NPs in the colloidal suspension, which corroborates the literature [33,34]. Chitosan acts as a reducing agent of gold as well as a stabilizer. The molecular weight and the degree of deacetylation (DD) of the chitosan present an important role in the nanoparticles hydrodynamic diameter [35]. In fact, Abrica-González et al. prepared $5 \mathrm{~nm}$ of chitosan AuNPs with a zeta potential of $43.3 \mathrm{mV}$, which is significantly different from the produced nanoparticles of this study. Chitosan with low Mw and DD allows the fabrication of stable chitosan nanoparticles with small diameters. The average of the functionalized CystAuNP hydrodynamic diameters is $54 \pm 9 \mathrm{~nm}$, a significant modification on the AuNPs size $(37 \mathrm{~nm})$ that could indicate the 
efficient functionalization of the nanoparticles. Their zeta potential is $-37 \mathrm{mV}$, suggesting good stability of the colloidal suspension.

The conjugation of ChAuNPs with EGCG was developed via carbodiimide-mediated cross-linking of carboxylic acids (Figure 2B). The hydrodynamic diameter is $125 \pm 13 \mathrm{~nm}$ and the average zeta potential is $36 \pm 6 \mathrm{mV}$ in water. ChAuNPs tend to form some stable aggregates due to the electrostatic attractive forces between amino groups in $\mathrm{Ch}$ and $\mathrm{AuCl}_{4^{-}}$ in suspension (zeta potential is $22 \pm 5 \mathrm{mV}$ ) [36,37].

A

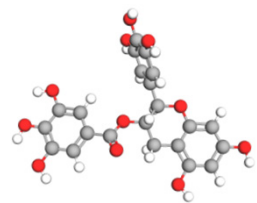

B
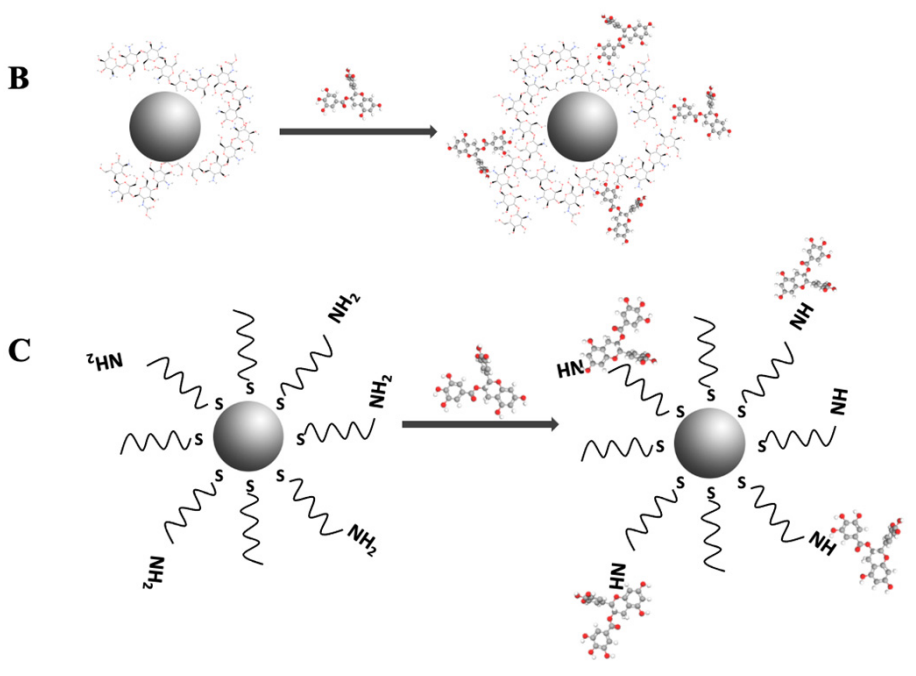

Figure 2. (A) Chemical structure of EGCG; (B) Scheme of nanoconjugate EGCG-ChAuNPs; (C) Scheme of nanoconjugate EGCG-CystAuNPs.

The polydispersity of the ChAuNPs occurs due to the degree of deacetylation of chitosan that promotes the increase in the PdI [24]. TEM analysis (Figure 3A) confirmed the spherical shape of the nanoparticles.
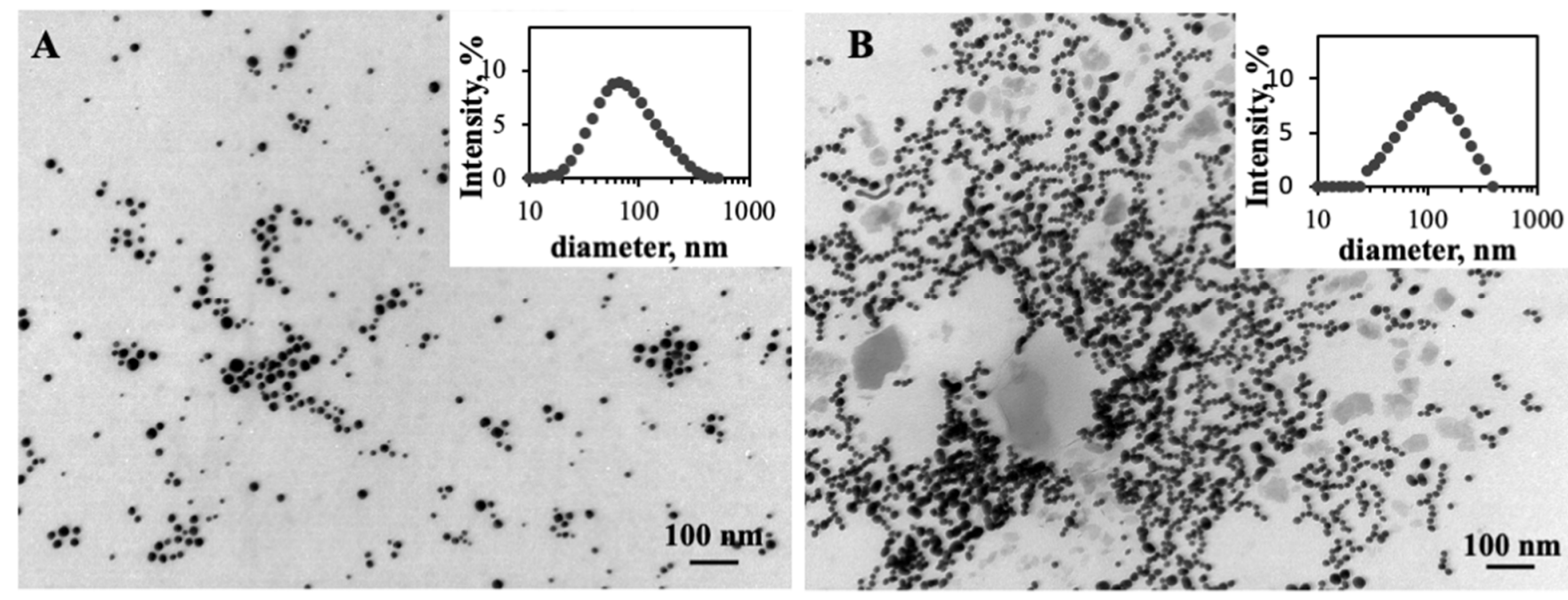

Figure 3. TEM image of: (A) EGCG-ChAuNPs; (B) EGCG-CystAuNPs. Scale bar is $100 \mathrm{~nm}$. 
The synthesized EGCG-CystAuNPs (Figure 3B) present $111 \pm 1 \mathrm{~nm}$ of diameter and a zeta potential of $-24 \pm 1 \mathrm{mV}$. The high AuNPs reactivity surface due to the EDC/NHSS coupling reaction might be the reason for the size increase.

Figure 4 displays the nanoparticles ATR-FTIR spectra. To confirm the chemical composition of the prepared nanosystems, ATR-FTIR spectra of EGCG, ChAuNPs, CystAuNPs, EGCG-ChAuNPs and EGCG-CystAuNPs were obtained.

A

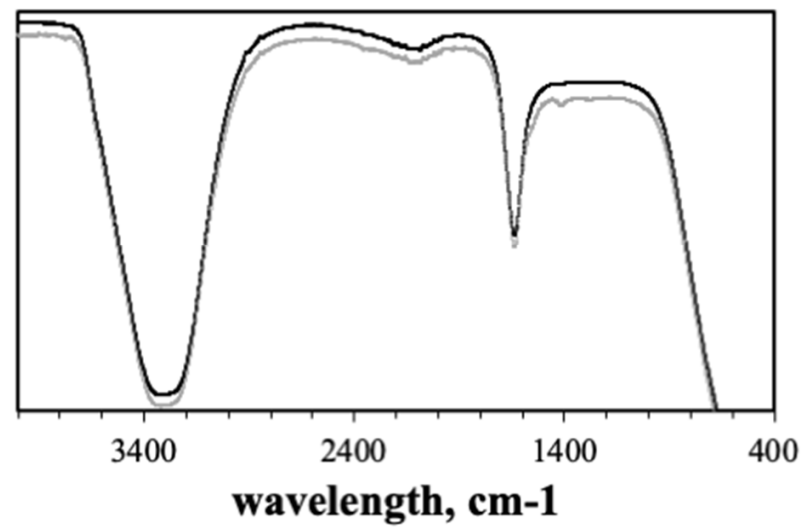

B

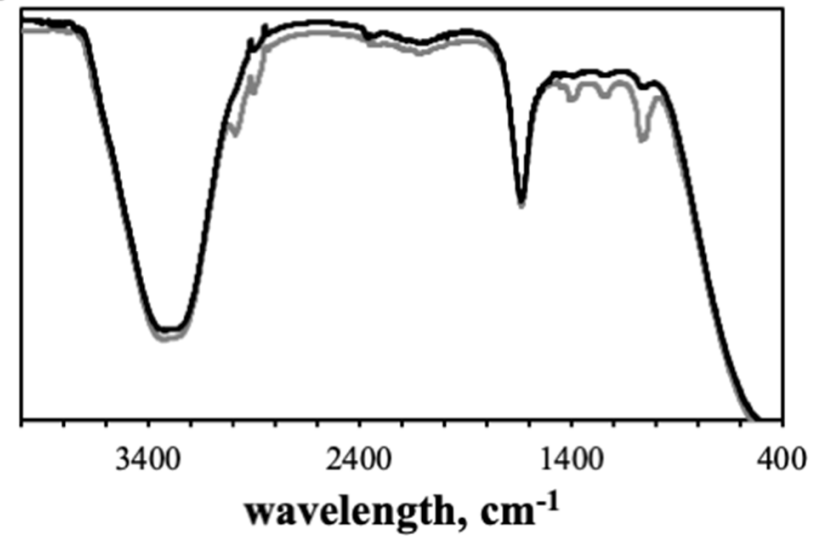

Figure 4. (A) FTIR spectra of ChAuNPs (grey line) and EGCG-ChAuNPs (black line). (B) FTIR spectra of CystAuNPs (grey line) and EGCG-CystAuNPs (black line). The spectra were shifted for a better visualization.

ChAuNPs spectrum demonstrates absorption bands at $3335 \mathrm{~cm}^{-1}$ (amine N-H symmetric vibration and $\mathrm{O}-\mathrm{H}$ group), at $2920 \mathrm{~cm}^{-1}$ from the $\mathrm{C}-\mathrm{H}$ group and at $1668 \mathrm{~cm}^{-1}$ and $1553 \mathrm{~cm}^{-1}$ from the $\mathrm{C}-\mathrm{O}$ stretching along with the $\mathrm{N}-\mathrm{H}$ deformation mode (acetylated amine and to free amine groups) (Figure 4A). The N-H bending suggests that the chitosan acts as stabilizer and reducing agent on the synthesis of AuNPs as reported by AbricaGonzález et al. [33]. The $\mathrm{CH}_{3}$ vibrations are presented at $1421 \mathrm{~cm}^{-1}$ and at $1296 \mathrm{~cm}^{-1}$; the $\mathrm{C}-\mathrm{N}$ bond is observed at $1301 \mathrm{~cm}^{-1}$. The spectra of conjugated EGCG-ChAuNPs (Figures 4A and S4A, black line) shows a peak at $3290 \mathrm{~cm}^{-1}$ assigned to the amine group. At $1553 \mathrm{~cm}^{-1}$ the $\mathrm{C}-\mathrm{O}$ stretching along with the $\mathrm{N}-\mathrm{H}$ deformation mode disappeared. A chemical modification by EDC/NHSS is suggested and it formed an amide bond covalently from ChAuNPs and polyphenol EGCG (at $1661 \mathrm{~cm}^{-1}, 1534 \mathrm{~cm}^{-1}$ and $1460 \mathrm{~cm}^{-1}$ visualized on Figure S3) [34]. On Figure 4B and Figure S4B, the CystAuNPs ATR-FTIR spectrum exhibited characteristic frequencies of $3653 \mathrm{~cm}^{-1}$ and $3671 \mathrm{~cm}^{-1}$ from the N-H stretch bond of primary amine. An intensive absorption band at $2988 \mathrm{~cm}^{-1}$ indicates $\mathrm{C}-\mathrm{H}$ of alkanes. The $2903 \mathrm{~cm}^{-1}$ wavenumber corresponds to a $\mathrm{C}-\mathrm{H}$ stretch of aldehyde. The peak at $1661 \mathrm{~cm}^{-1}$ and $2051 \mathrm{~cm}^{-1}$ might indicate the salt formed by CSH with $\mathrm{HCl}\left(-\mathrm{NH}_{3}^{+}\right)$. The $1409 \mathrm{~cm}^{-1}$ band corresponds to the strong symmetric vibration of the $v C=O\left(1245 \mathrm{~cm}^{-1}\right)$, which indicates the stretching vibration of $\mathrm{C}-\mathrm{N}$ bond. In the spectrum of EGCG-CystAuNPs, the wavenumber $1542 \mathrm{~cm}^{-1}$ indicates the $\mathrm{N}-\mathrm{H}$ bond from amide linked by alcohol group of polyphenol EGCG and primary amine of the nanoparticles, via EDC/NHSS coupling reaction (Figure S4B) [23]. The C-H group of aromatic ring at $845 \mathrm{~cm}^{-1}$ was observed.

EGCG conjugation efficiency was determined for both nanosystems, by UV-Vis spectroscopy. A total of $60 \pm 2 \%$ and $78 \pm 1.3 \%$ values of EGCG encapsulation efficiency achieved to EGCG-ChAuNPs and EGCG-CystAuNPs, respectively.

The antioxidant activity of EGCG conjugated with ChAuNPs and CystAuNPs was studied and presented in Table 2 . The free radical scavenging activity of the produced nanosystems was assessed by the ABTS method. 
Table 2. ABTS radical scavenging activity of ChNPs, CystAuNPs, EGCG-ChAuNPs and EGCGCystAuNPs.

\begin{tabular}{ccccc}
\hline & & \multicolumn{3}{c}{ Antioxidant Activity (mM) } \\
Wavelength $(\mathrm{nm})$ & AuChNPs & CystAuNPs & EGCG-ChAuNPs & EGCG-CystAuNPs \\
\hline 750 & - & 1.8 & 2.5 & 3.5 \\
\hline
\end{tabular}

According to the results, EGCG-ChAuNPs and EGCG-CystAuNPs present high capacity to scavenge the ABTS generated in aqueous phase. This suggested that EGCG could be protected by the nanoparticles and the antioxidant activity of EGCG was preserved. Additionally, the antioxidant capacity of the EGCG might be improved by the conjugation with AuNPs.

The stability of EGCGChAuNPs and EGCGCystAuNPs was evaluated in terms of hydrodynamic diameter and zeta potential, in pH 5.3 and pH 7.4 at $37^{\circ} \mathrm{C}$, in the dark for 60 days. Data suggested stable EGCGChAuNPs for $24 \mathrm{~h}$ and for 7 days at $\mathrm{pH} 7.4$ and $\mathrm{pH} 5.3$, respectively. EGCGCystAuNPs presents higher stability at $\mathrm{pH} 7.4$ and $37^{\circ} \mathrm{C}$ (60 days) than at $\mathrm{pH} 5.3$ and $37^{\circ} \mathrm{C}$ ( 2 days), suggesting that the high electrostatic repulsion is responsible for the maintenance of the size of the nanoparticles.

The in vitro release kinetics from nanoconjugates were performed, using dialysis membranes in PBS solution at $37^{\circ} \mathrm{C}$ and $\mathrm{pH}$ 7.4. The conjugated EGCG exhibits a fast release from ChAuNPs (Figure 5A). This fact might occur due to part of EGCG being absorbed in the nanoparticles surface.

A

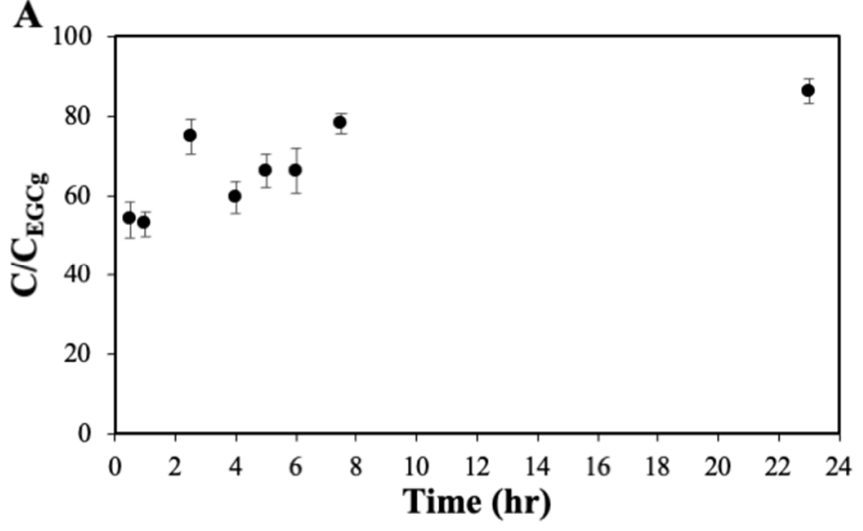

B

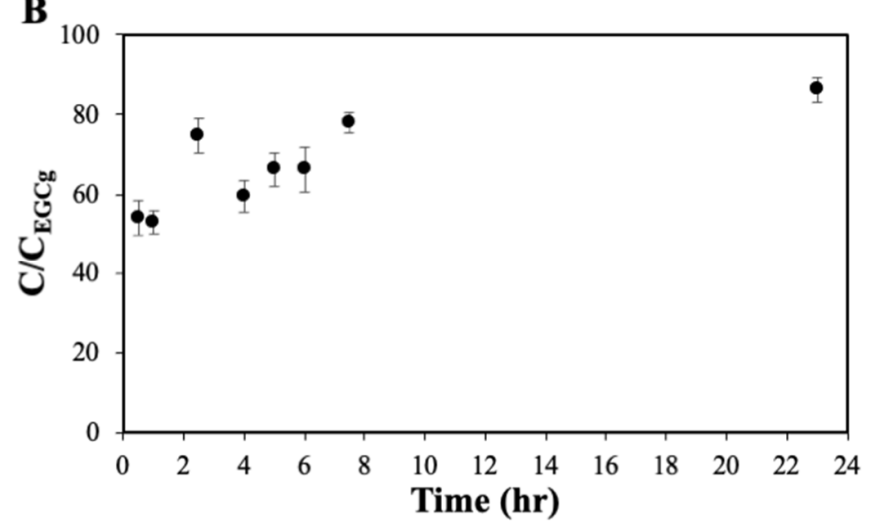

Figure 5. In vitro release profiles of (A) EGCG-ChAuNPs and (B) EGCG-CystAuNPs at pH 7.4 (in PBS $0.01 \mathrm{M}$ ) at $37^{\circ} \mathrm{C}$. $\mathrm{C}_{\text {EGCG }}$ corresponds to the total amount of EGCG added.

In fact, after $30 \mathrm{~min}$, 54\% of EGCG was released from EGC-ChAuNPs nanoconjugates (Figure $5 \mathrm{~A}$ ). In $8 \mathrm{~h}, 52 \%$ of the EGCG was released from EGCG-CystAuNPs (Figure 5B). Comparing both nanoconjugates, after $24 \mathrm{~h}$ of incubation, $86.2 \%$ and $60.9 \%$ of the EGCG was released from ChAuNPs and CystAuNPs, respectively. These data correspond to 0.038 and $0.041 \mathrm{mM}$ of EGCG concentration in CystAuNPs and ChAuNPs suspensions, respectively. The complete amount of catechin was achieved after $48 \mathrm{~h}$.

The cytotoxicity of ChAuNPs and CystAuNPs to BxPC3 cells was evaluated by the SRB method. Figure 6 displays the cell growth results of the cancer cells after $24 \mathrm{~h}$ of incubation with the nanoparticles alone. Figure 5 shows that, for concentrations up to $1 \mathrm{nM}$, CystAuNPs do not have effect on the cell growth of BxPC3 cells. For ChAuNPs, concentrations up to $2 \mathrm{nM}$ the same effect was verified. For concentrations above $0.2 \mathrm{nM}$ until $1 \mathrm{nM}$, ChAuNPs present $10 \%$ of toxicity on this type of cancer cells. 


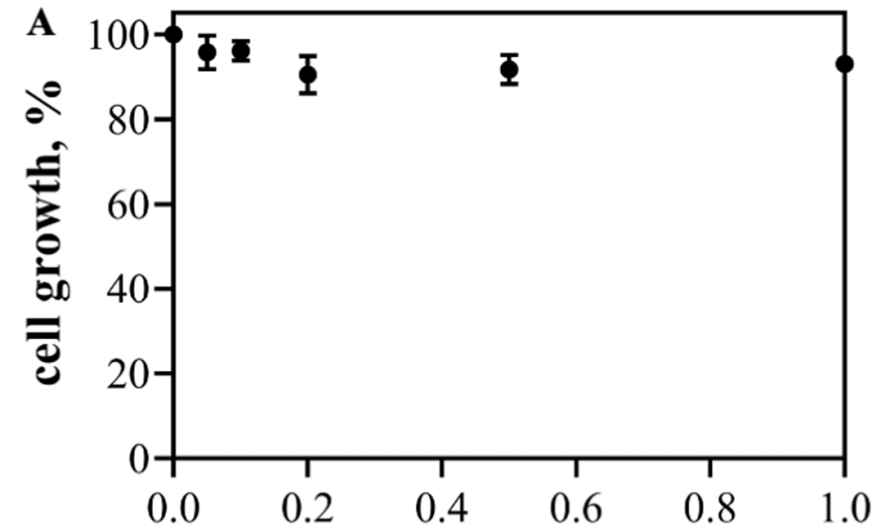

Concentration of ChAuNPs, nM

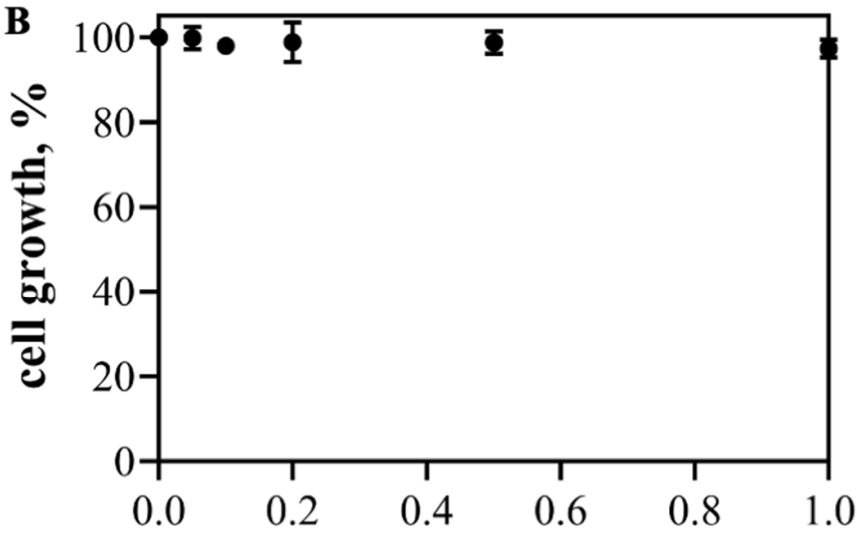

Concentration of CystAuNPs, nM

Figure 6. Effect of ChAuNPs (A) and CystAuNPs (B) on the cell growth of pancreatic cancer BxPC3 cells.

The cytotoxic effect of EGCG and the nanosystems (EGCG-ChAuNPs and CystAuNPs) was studied against the same cancer cell line, the BxPC3 cells (Figure 7).

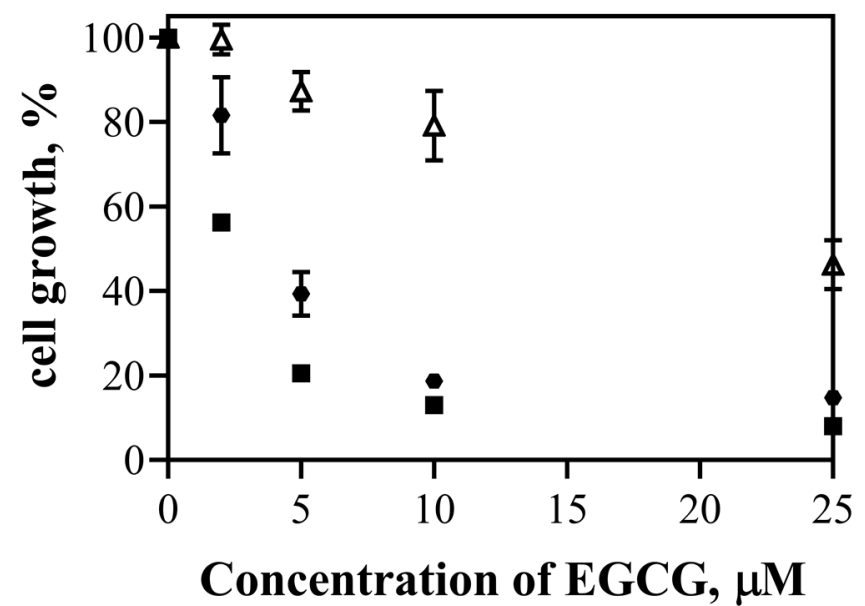

Figure 7. Effect of free EGCG $(\Delta)$, EGCG-AuChNPs $(\boldsymbol{\square})$ and EGCG-CysAuNPs $(\bullet)$ on the growth of pancreatic cancer (BxPC3 cells).

The cell growth of BxPC3 incubated with EGCG alone was found to be in the range of $99.6-46.3 \%$ for the EGCG concentration of 2.0-25.0 $\mu \mathrm{M}$. For the same concentrations, it presented cell growth in the range of $56.2-8.0 \%$ and $81.6-14.7 \%$ when incubated with EGCGChAuNPs and EGCG-CystAuNPs, respectively. These values demonstrated a significant effectiveness of both nanoconjugates comparing with EGCG alone, as visualized by $\mathrm{IC}_{50}$ in Table 3. In particular, for an EGCG concentration of 5 and $10 \mu \mathrm{M}$, the cytotoxicity of conjugated EGCG (both nanosystems) was significantly higher than EGCG alone. The cell growth of BxPC3 decreases from $87.3 \%$ incubated with $5 \mu \mathrm{M}$ EGCG alone, to $20.5 \%$ and $39.3 \%$ when incubated with EGCG-ChAuNPs and EGCG-CystAuNPs, respectively. These results are in accordance with the achieved antioxidant activity of the nanoconjugates as well as the in vitro release profiles of the EGCG-ChAuNPs and EGCG-CystAuNPs. In fact, the nanoconjugates presented a high antioxidant capacity that could be reliable for the decrease in the BxPC3 cell growth. Additionally, the data corroborated the literature and the enhancement of the chemical activity of EGCG when conjugated on the gold nanosurface might occur [17]. 
Table 3. Half maximal inhibitory concentration $\left(\mathrm{IC}_{50}\right)$ values on $\mathrm{BxPC} 3$ cells.

\begin{tabular}{cccc}
\hline & Free EGCG & EGCG-ChAuNPs & EGCG-CystAuNPs \\
\hline $\mathrm{IC}_{50, \mu \mathrm{M}}$ & $23 \pm 3$ & $2.22 \pm 0.02$ & $3.7 \pm 0.2$ \\
\hline
\end{tabular}

Conjugated EGCG significantly induced apoptosis in BxPC3 as evidenced by the increase in caspase- 3 activity when compared with non-treated $\mathrm{BxPC} 3$ cells and cells treated with ChAuNPs and CystAuNPs at $0.1 \mathrm{nM}$ of concentration (Figure 8). For EGCG-ChAuNPs, this evidence is more pronounced.

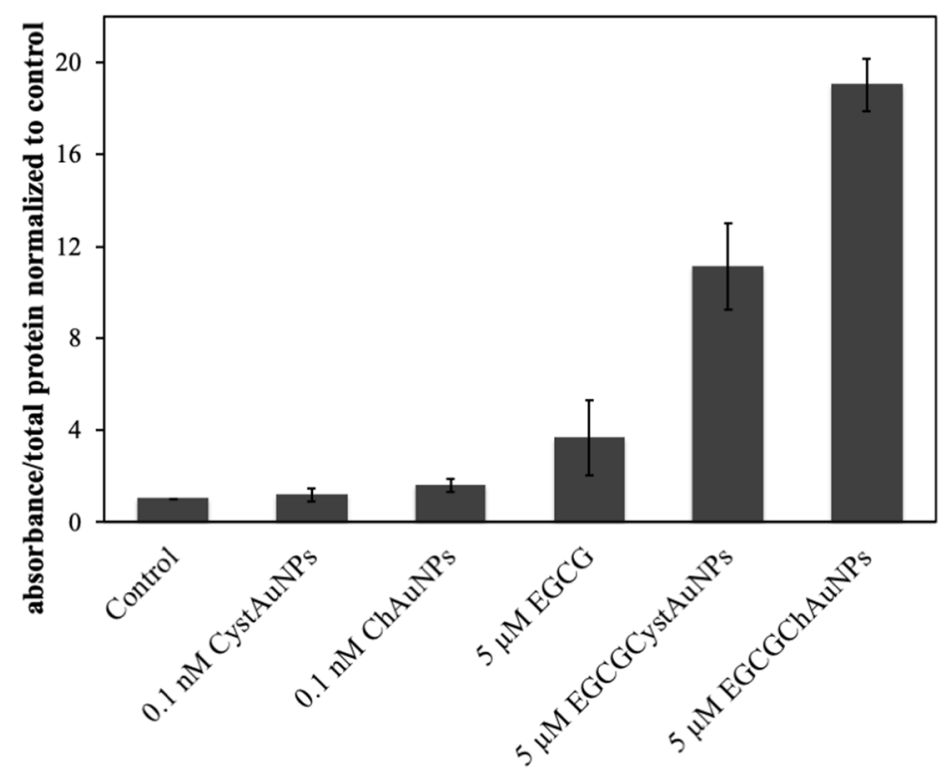

Figure 8. Activation of caspase-3 in BxPC3 cells exposed for $24 \mathrm{~h}$ to free EGCG, EGCG-CystAuNPs, EGCG-ChAuNPs, CystAuNPs and ChAuNPs.

\section{Conclusions}

This work proposes two methodologies to prepare nanocarriers based on gold nanoparticles decorated with EGCG. We demonstrated that the EGCG conjugates nanosystems enhance the inhibition of BxPC3 cell growth than EGCG alone. It is possible to decrease the EGCG-ChAuNPs and EGCG-CystAuNPs concentration 10.5 and 6.4 times to have the same effect as the EGCG alone. This effect is more pronounced by the positive charged EGCG-ChAuNPs. This fact might promote the presence of attractive forces, resulting in an enhanced accumulation and BXPC3 cell uptake.

In summary, these results suggest that the effectiveness of EGCG conjugated NPs for cancer therapy increase the antioxidant efficacy and, therefore, the cell growth inhibition. This study could provide promising delivery systems with an encapsulation efficiency above $60 \%$ and the possibility of scaling up the process for the pancreatic cancer treatment. The nanoparticles were nontoxic to BxPC3 cancer cells proposing that the AuNPs could be produced as a chemopreventive vector. In vitro assays showed that EGCG-ChAuNP and EGCG-CystAuNP nanoconjugates present 50\% inhibitory concentration 10.4 and 6.2 times lower than the EGCG alone, respectively. To have a better understanding of the concept based on these systems, other type of cancer cells and even normal cell lines should be tested.

Supplementary Materials: The following are available online at https:/ / www.mdpi.com/article/ 10.3390/pharmaceutics14030491/s1, Figure S1: UV-vis absorption spectra of: (A) EGCG-ChAuNPs and (B) EGCG-CystAuNPs, Figure S2: In vitro release spectra of (A) EGCG-ChAuNPs and (B) EGCGCystAuNPs at $\mathrm{pH} 7.4\left(\right.$ in PBS $0.01 \mathrm{M}$ ) at $37^{\circ} \mathrm{C}$. $\bullet$ corresponds to the lowest release data; $\bullet$ corresponds to the highest release data. CEGCG corresponds to the total amount of EGCG added, Figure S3: 
FTIR spectra of EGCG. The spectra were shifted for a better visualization, Figure S4: FTIR spectra of: (A) EGCG-ChAuNPs and (B) EGCG-CystAuNPs. The spectra were shifted for a better visualization. The final spectra correspond to the subtraction of deionized water FTIR spectrum to the nanoconjugates FTIR spectra, Figure S5: Effect of ChAuNPs (A) and CystAuNPs (B) on the cell growth of BxPC3 cells. $-\mathbf{\Delta}$ - and - $\mathbf{\Delta}$ - correspond to the lowest and highest cell growth values, respectively, Figure S6: Effect of free EGCG ( $\boldsymbol{\Delta}$ and $\boldsymbol{\Delta})$, EGCG-AuChNPs ( $\boldsymbol{\square}$ and $\boldsymbol{\square})$ and EGCG-CysAuNPs $(\bullet$ and $\bullet$ ) on the growth of pancreatic cancer. The grey and black symbol correspond to the lowest and highest cell growth data, respectively, Figure S7: Activation of caspase-3 in pancreatic cancer cells exposed for $24 \mathrm{~h}$ to free EGCG, EGCG-CystAuNPs, EGCG-ChAuNPs, CystAuNPs and ChAuNPs. Grey and black column correspond to the lowest and highest absorbance/total protein normalized to control, respectively.

Author Contributions: Conceptualization, S.C.C., M.d.C.P. and M.A.N.C.; data Curation, S.C.C.; formal analysis, S.C.C. and L.C.; funding acquisition, M.A.N.C.; investigation, S.C.C., L.C., M.d.C.P. and M.A.N.C.; methodology, L.C. and S.C.C.; software, L.C. and S.C.C.; supervision, S.C.C., M.d.C.P. and M.A.N.C.; validation, L.C. and S.C.C.; writing-original draft preparation, L.C. and S.C.C.; writing-review and editing, S.C.C., L.C., M.d.C.P. and M.A.N.C. All authors have read and agreed to the published version of the manuscript.

Funding: This work was financially supported by LA/P/0045/2020 (ALiCE), UIDB/00511/2020 and UIDP/00511/2020 (LEPABE), funded by national funds through FCT/MCTES (PIDDAC).

Acknowledgments: We would like to thank Aleksandra Wietecka, Erasmus student from the Faculty of Chemistry Poznan of Adam Mickiewicz University (Poznań), for the collaboration with physicochemical analysis of the nanosystems. We would like to thank Rui Fernandes for the collaboration with TEM analysis.

Conflicts of Interest: The authors have no other relevant affiliation or financial involvement with any organization or entity with financial interest in or financial conflict manuscript apart from those disclosed.

\section{References}

1. Gao, Q.; Zhang, J.; Gao, J.; Zhang, Z.; Zhu, H.; Wang, D. Gold Nanoparticles in Cancer Theranostics. Front. Bioeng. Biotechnol. 2021, 9, 221. [CrossRef] [PubMed]

2. Coelho, S.C.; Reis, D.P.; Pereira, M.C.; Coelho, M.A.N. Gold Nanoparticles for Targeting Varlitinib to Human Pancreatic Cancer Cells. Pharmaceutics 2018, 10, 91. [CrossRef] [PubMed]

3. Wang, S.; Lu, G. Aplications of Gold Nanoparticles in Cancer Imaging and Treatment. In Applications of Gold Nanoparticles in Cancer Imaging and Treatment, Noble and Precious Metals_Properties, Nanoscale Effects and Applications; Seehra, M.S., Ed.; IntechOpen: London, UK, 2017.

4. Parveen, S.; Misra, R.; Sahoo, S.K. Nanoparticles: A boon to drug delivery, therapeutics, diagnostics and imaging. Nanomed. Nanotechnol. Biol. Med. 2012, 8, 147-166. [CrossRef] [PubMed]

5. Rahmani, A.H.; Al Shabrmi, F.M.; Allemailem, K.S.; Aly, S.M.; Khan, M.A. Implications of Green Tea and Its Constituents in the Prevention of Cancer via the Modulation of Cell Signalling Pathway. BioMed Res. Int. 2015, 2015, 925640. [CrossRef] [PubMed]

6. Safwat, M.A.; Kandil, B.A.; Elblbesy, M.A.; Soliman, G.M.; Eleraky, N.E. Epigallocatechin-3-Gallate-Loaded Gold Nanoparticles: Preparation and Evaluation of Anticancer Efficacy in Ehrlich Tumor-Bearing Mice. Pharmaceuticals 2020, 13, 254. [CrossRef] [PubMed]

7. Rao, P.V.; Nallappan, D.; Madhavi, K.; Rahman, S.; Jun Wei, L.; Gan, S.H. Phytochemicals and Biogenic Metallic Nanoparticles as Anticancer Agents. Oxid. Med. Cell. Longev. 2016, 2016, 3685671. [CrossRef]

8. Li, K.; Teng, C.; Min, Q. Advanced Nanovehicles-Enabled Delivery Systems of Epigallocatechin Gallate for Cancer Therapy. Front. Chem. 2020, 8, 874. [CrossRef]

9. Choi, Y.; Choi, M.-J.; Cha, S.-H.; Kim, Y.S.; Cho, S.; Park, Y. Catechin-capped gold nanoparticles: Green synthesis, characterization, and catalytic activity toward 4-nitrophenol reduction. Nanoscale Res. Lett. 2014, 9, 103. [CrossRef]

10. Sanna, V.; Pala, N.; Dessì, G.; Manconi, P.; Mariani, A.; Dedola, S.; Rassu, M.; Crosio, C.; Iaccarino, C.; Sechi, M. Single-step green synthesis and characterization of gold-conjugated polyphenol nanoparticles with antioxidant and biological activities. Int. J. Nanomed. 2014, 9, 4935-4951. [CrossRef]

11. Mostafa, S.M.; Gamal-Eldeen, A.M.; Maksoud, N.A.E.; Fahmi, A.A. Epigallocatechin gallate-capped gold nanoparticles enhanced the tumor suppressors let-7a and miR-34a in hepatocellular carcinoma cells. An. Acad. Bras. Ciências 2020, 92, e20200574. [CrossRef]

12. Rocha, S.; Generalov, R.; Pereira, M.D.C.; Peres, I.; Juzenas, P.; Coelho, M.A.N. Epigallocatechin gallate-loaded polysaccharide nanoparticles for prostate cancer chemoprevention. Nanomedicine 2010, 6, 79-87. [CrossRef] [PubMed] 
13. Xia, Q.; Huang, J.; Feng, Q.; Chen, X.; Liu, X.; Li, X.; Zhang, T.; Xiao, S.; Li, H.; Zhong, Z.; et al. Size- and cell type-dependent cellular uptake, cytotoxicity and in vivo distribution of gold nanoparticles. Int. J. Nanomed. 2019, 14, 6957-6970. [CrossRef] [PubMed]

14. Foroozandeh, P.; Aziz, A.A. Insight into Cellular Uptake and Intracellular Trafficking of Nanoparticles. Nanoscale Res. Lett. 2018, 13, 339. [CrossRef] [PubMed]

15. Kimling, J.; Maier, M.; Okenve, B.; Kotaidis, V.; Ballot, H.; Plech, A. Turkevich Method for Gold Nanoparticle Synthesis Revisited. J. Phys. Chem. B 2006, 110, 15700-15707. [CrossRef] [PubMed]

16. Coelho, S.C.; Almeida, G.M.; Pereira, M.C.; Santos-Silva, F.; Coelho, M.A.N. Functionalized gold nanoparticles improve afatinib delivery into cancer cells. Expert Opin. Drug Deliv. 2016, 13, 133-141. [CrossRef]

17. Boyles, M.S.P.; Kristl, T.; Andosch, A.; Zimmermann, M.; Tran, N.; Casals, E.; Himly, M.; Puntes, V.; Huber, C.G.; LützMeindl, U.; et al. Chitosan functionalisation of gold nanoparticles encourages particle uptake and induces cytotoxicity and proinflammatory conditions in phagocytic cells, as well as enhancing particle interactions with serum components. J. Nanobiotechnol. 2015, 13, 84. [CrossRef]

18. Aibani, N.; Rai, R.; Patel, P.; Cuddihy, G.; Wasan, E.K. Chitosan Nanoparticles at the Biological Interface: Implications for Drug Delivery. Pharmaceutics 2021, 13, 1686. [CrossRef]

19. Fujisawa, T.; Rubin, B.; Suzuki, A.; Patel, P.S.; Gahl, W.A.; Joshi, B.H.; Puri, R.K. Cysteamine suppresses invasion, metastasis and prolongs survival by inhibiting matrix metalloproteinases in a mouse model of human pancreatic cancer. PLoS ONE 2012, 7, e34437. [CrossRef]

20. Rubin, B.; Gilbert, M.; Jung, J. Use of Cysteamine and Derivatives Thereof to Supress Tumor Metastases. U.S. Patent 20210290569, 2 June 2021.

21. Sulaiman, G.M.; Waheeb, H.M.; Jabir, M.S.; Khazaal, S.H.; Dewir, Y.H.; Naidoo, Y. Hesperidin Loaded on Gold Nanoparticles as a Drug Delivery System for a Successful Biocompatible, Anti-Cancer, Anti-Inflammatory and Phagocytosis Inducer Model. Sci. Rep. 2020, 10, 9362. [CrossRef]

22. Leu, J.-G.; Chen, S.-A.; Chen, H.-M.; Wu, W.-M.; Hung, C.-F.; Yao, Y.-D.; Tu, C.-S.; Liang, Y.-J. The effects of gold nanoparticles in wound healing with antioxidant epigallocatechin gallate and $\alpha$-lipoic acid. Nanomedicine 2011, 8, 767-775. [CrossRef]

23. Cheung, R.C.F.; Ng, T.B.; Wong, J.H.; Chan, W.Y. Chitosan: An Update on Potential Biomedical and Pharmaceutical Applications. Mar. Drugs 2015, 13, 5156-5186. [CrossRef] [PubMed]

24. Li, W.; Cao, Z.; Liu, R.; Liu, L.; Li, H.; Li, X.; Chen, Y.; Lu, C.; Liu, Y. AuNPs as an important inorganic nanoparticle applied in drug carrier systems. Artif. Cells Nanomed. Biotechnol. 2019, 47, 4222-4233. [CrossRef] [PubMed]

25. Bhattarai, S.R.; Kc, R.B.; Aryal, S.; Bhattarai, N.; Kim, S.Y.; Yi, H.K.; Hwang, P.H.; Kim, H.Y. Hydrophobically modified chitosan/gold nanoparticles for DNA delivery. J. Nanoparticle Res. 2008, 10, 151-162. [CrossRef]

26. Elgadir, M.A.; Uddin, S.; Ferdosh, S.; Adam, A.; Chowdhury, A.J.K.; Sarker, Z.I. Impact of chitosan composites and chitosan nanoparticle composites on various drug delivery systems: A review. J. Food Drug Anal. 2015, 23, 619-629. [CrossRef]

27. Safer, A.-M.; Leporatti, S.; Jose, J.; Soliman, M.S. Conjugation Of EGCG And Chitosan NPs As A Novel Nano-Drug Delivery System. Int. J. Nanomed. 2019, 14, 8033-8046. [CrossRef] [PubMed]

28. Wan, X.M.; Zheng, F.; Zhang, L.; Miao, Y.Y.; Man, N.; Wen, L.P. Autophagy-mediated chemosensitization by cysteamine in cancer cells. Int. J. Cancer 2011, 129, 1087-1095. [CrossRef] [PubMed]

29. Inano, H.; Onoda, M.; Suzuki, K.; Kobayashi, H.; Wakabayashi, K. Inhibitory Effects of WR-2721 and Cysteamine on Tumor Initiation in Mammary Glands of Pregnant Rats by Radiation. Radiat. Res. 2000, 153, 68-74. [CrossRef]

30. Huang, H.; Yang, X. Synthesis of Chitosan-Stabilized Gold Nanoparticles in the Absence/Presence of Tripolyphosphate. Biomacromolecules 2004, 5, 2340-2346. [CrossRef]

31. Baptista, P.; Doria, G.; Henriques, D.; Pereira, E.; Franco, R. Colorimetric detection of eukaryotic gene expression with DNAderivatized gold nanoparticles. J. Biotechnol. 2005, 119, 111-117. [CrossRef]

32. Coelho, S.C.; Rocha, S.; Juzenas, P.; Sampaio, P.; Almeida, G.M.; Silva, F.S.; Pereira, M.C.; Coelho, M.A.N. Gold nanoparticle delivery-enhanced proteasome inhibitor effect in adenocarcinoma cells. Expert Opin. Drug Deliv. 2013, 10, 1345-1352. [CrossRef]

33. Abrica-González, P.; Zamora-Justo, J.A.; Sotelo-López, A.; Vázquez-Martínez, G.R.; Balderas-López, J.A.; Muñoz-Diosdado, A.; Ibáñez-Hernández, M. Gold nanoparticles with chitosan, N-acylated chitosan, and chitosan oligosaccharide as DNA carriers. Nanoscale Res. Lett. 2019, 14, 258. [CrossRef] [PubMed]

34. Mohan, C.O.; Gunasekaran, S.; Ravishankar, C.N. Chitosan-capped gold nanoparticles for indicating temperature abuse in frozen stored products. NPJ Sci. Food 2019, 3, 2. [CrossRef] [PubMed]

35. Mun, S.; Decker, E.A.; McClements, D.J. Effect of molecular weight and degree of deacetylation of chitosan on the formation of oil-in-water emulsions stabilized by surfactant-chitosan membranes. J. Colloid Interface Sci. 2006, 296, 581-590. [CrossRef] [PubMed]

36. Wu, L.; Shi, C.; Tian, L.; Zhu, J. A One-Pot Method to Prepare Gold Nanoparticle Chains with Chitosan. J. Phys. Chem. C 2008, 112, 319-323. [CrossRef]

37. Jiang, C.; Zhu, J.; Li, Z.; Luo, J.; Wang, J.; Sun, Y. Chitosan-gold nanoparticles as peroxidase mimic and their application in glucose detection in serum. RSC Adv. 2017, 7, 44463-44469. [CrossRef] 